Functional residual capacity, inspiratory capacity, vital capacity, forced vital capacity, airways resistance, residual volume and gas mixing efficiency were within normal limits and did not change. Only peak expiratory flow showed significant improvement after four weeks (7.79-8.28 I.sec).

Blood volumes, red-cell volumes and haemoglobin were unaffected by this relatively short training programme. Two cardiac enzymes, AST and ALT were within normal limits before training and did not change. The third, CK, was $168 \%$ higher than the normal range of 5-40 u.l. before training (107.1 u.l. $\mp$ 72.3), fell significantly after eight weeks (64.7 u.I. $\mp 36.9$ ) but was still $62 \%$ above normal limits. Blood lactate at standard work was also lower following training $(5.37 \mathrm{~m} . \mathrm{mol} . \mathrm{l}$ $\mp$ 2.29-4.14 m.mol.I. $\mp 1.70)$ but 2-3 d-PG remained unchanged.

Raised levels of CK reflect skeletal muscle injury. The high levels noted before training may be the result of a sharp increase in physical activity experienced by the students as they continued their physical education programme after the long summer vacation. The importance of above normal levels of CK is not yet clear and further work is needed to support the findings reported here and test the hypothesis that CK may be a simple index of straining rather than training.

This work was supported by a grant from WSIHE Research Committee.

\section{THE EFFECT OF GRADED AND EXHAUSTIVE PHYSICAL EXERCISE ON VASOPRESSIN RESPONSE IN NORMAL, HEALTHY VOLUNTEERS}

\author{
M. S. El-Sayed*, B. Davies* and Prof. D. B. Morgan
}

*Human Performance Laboratory, University of Salford

* Dept. of Chemical Pathology, University of Leeds

The vasopressin response to steady-state and exhaustive exercise was investigated in 16 normal, healthy volunteers. The maximum functional capacity test comprised the basis for calculation of the relative intensities corresponding to heart rates equivalent to $40 \%$ and $70 \%$ $\mathrm{VO}_{2}$ max. The steady-state exercise tests (i.e. $40 \%$, $70 \%$ and $70 \%$ + heat for 15 minutes) were assigned to the subjects using a latin square design, with seven days intervening between tests. Following $\mathbf{3 0}$ minutes of supine rest, $25 \mathrm{ml}$ of venous blood was removed using a two-syringe technique. Immediately after the cessation of exercise, a second blood sample was removed $(25$ $\mathrm{mI)}$ for subsequent analysis of vasopressin using RIA.

Results

Non-significant ( $p>0.05$ ) inter- or intra-individual variations in the resting level of vasopressin were estab- lished over the four testing occasions, the mean values being $0.97,1.28,0.99$ and $1.04 \mathrm{ng} / \mathrm{L}$ respectively. A significant difference $(p<0.05)$ was found in the mean vasopressin responses to graded and exhaustive exercise at $70 \%$ (x 3.22), $70 \%$ + heat (x 3.79) and 100\% ( $x$ 11.65), with a non-significant vasopressin response at $40 \%$ (x 1.93). When the vasopressin was measured following three repeated exercise tests at $70 \% \mathrm{VO}_{2}$ max, the reproducibility was within $\pm 10 \%$. Accordingly, it is concluded that physical exercise induces a significant increase in the level of vasopressin, probably as a contributing factor in the regulation of plasma volume.

\section{A TECHNIQUE FOR MONITORING THE EFFECTS OF TRAINING ON SWIMMING EFFICIENCY}

\author{
J. Watkins, PhD* and J. Park, DPE** \\ *Lecturer in Physical Education, SSPE, \\ Jordanhill College of Education, Glasgow \\ * *Head Coach, Paisley Swimming Club, Glasgow
}

Most of the studies of swimming efficiency in man have involved the use of expensive specialised equipment (Di Prampero et al, 1974; Kemper et al, 1976). These studies have provided considerable information concerning the physiology and biomechanics of swimming but there does not seem to have been much spin-off for the club coach in terms of methods of assessing swimming efficiency and, therefore, methods of evaluating the effects of different training programmes (Costill et al, 1980). The present paper outlines the development of a technique for monitoring the effects of training on swimming efficiency for use with welltrained swimmers. The technique is based on the relationship between heart rate and swimming speed which has been shown to be linear for well-trained swimmers (Treffene, 1978). Each swimmer is required to swim three trials of $200 \mathrm{~m}$ at speeds which correspond approximately to moderate, fast and all-out efforts. During each trial the elapsed time between push-off and touch is recorded to the nearest tenth of a second and the heart rate of the swimmer is assessed at the end of each trial by taking a pulse count during the period 0-15 seconds of recovery. A least squares regression line is calculated from the speed:heart rate data. The gradient of the line and the predicted speed at a heart rate of 190 beats per minute (S190) are used as measures of swimming efficiency. With a group of fifteen welltrained swimmers, including five current internationalists, the effects of endurance swimming training (rather than sprint training or land conditioning) is to increase both the gradient and the S190 which are reflected in performance times in competitive events.

\section{References}

Costill, D., Sharp, R. and Troup, J., 1980 "Muscle strength: contributions to speed swimming". Swimming World 21 (2): 29-34. 